\title{
Propagation of the Irregularity of a Microdifferential System
}

\author{
By \\ Teresa Monteiro FeRnAndES*
}

\begin{abstract}
We construct the functor of microlocal analytic irregularity $I \mu$ hom $\left(\cdot, \mathcal{O}_{X}\right)$ which gives a natural third term of a distinguished triangle associated to the transformation $t \mu \operatorname{hom}\left(\cdot, \mathcal{O}_{X}\right) \rightarrow \mu \operatorname{hom}\left(\cdot, \mathcal{O}_{X}\right)$ of functors on the derived category of $\mathbb{R}$-constructible sheaves. When restricting to $\mathbb{C}$-constructible objects we prove that the microlocal irregularity of a microdifferential system propagates along non 1-microcharacteristic directions, as a consequence of the propagation for $t \mu \operatorname{hom}\left(\cdot, \mathcal{O}_{X}\right)$ and $\mu \operatorname{hom}\left(\cdot, \mathcal{O}_{X}\right)$.
\end{abstract}

\section{Introduction}

In this paper we treat a problem posed by P. Schapira : to show that the 1-microcharacteristic variety of a microdifferential system $\mathcal{M}$ along an involutive submanifold $V$ contains the microsupport of its solutions in the sheaves $t \mu \operatorname{hom}\left(F, \mathcal{O}_{X}\right), \mu \operatorname{hom}\left(F, \mathcal{O}_{X}\right)$, whenever the microsupport of the $\mathbb{R}$ constructible complex $F$ is contained in $V$. Here $\mathcal{O}_{X}$ denotes the sheaf of holomorphic functions on the complex manifold $X$. In other words, the microlocal $F$-irregularity of $\mathcal{M}$ propagates along the non 1-microcharacteristic directions. Let us recall that $\mu \operatorname{hom}\left(\cdot, \mathcal{O}_{X}\right)$ and $t \mu \operatorname{hom}\left(\cdot, \mathcal{O}_{X}\right)$ are the microlocalized of the functors $\mathbb{R} \mathcal{H} \operatorname{om}\left(\cdot, \mathcal{O}_{X}\right)$ and $t \mathcal{H o m}\left(\cdot, \mathcal{O}_{X}\right)$, and were respectively introduced by Kashiwara-Schapira (cf. [K-S3]) and Andronikof (cf. [A]).

Communicated by M. Kashiwara, May 31, 1999. Revised December 13, 1999 and February $21,2000$.

2000 Mathematics Subject Classification(s): 58J15, 58J47, 35A21, 35A27.

Key words: Microsupport, irregularity, 1-microcharacteristic variety, microdifferential systems.

Work supported by FCT, project PRAXIS/2/2.1/MAT/125/94, FEDER and PRAXIS XXI.

* Departamento de Matemática da Universidade de Lisboa Centro de Álgebra/UL, Complexo II 2, Av. Prof. Gama Pinto 1649-003 LISBOA Codex Portugal.

e-mail: tmf@ptmat.lmc.fc.ul.pt 
At the present status of microlocal analysis, it is not clear if the sheaf $\mathcal{E}_{X}$ of microdifferential operators acts on $\mu$ hom $\left(F, \mathcal{O}_{X}\right)$ and $t \mu$ hom $\left(F, \mathcal{O}_{X}\right)$ but only on its cohomology; however, the work developped by Kashiwara and Schapira ([K-S5]) seems to indicate a positive answer. In this paper, we will treat the $\mathbb{C}$-constructible case, and $F$ is supposed to be perverse.

When $F=\mathbb{C}_{Y}$ for a complex $d$-codimensional submanifold, one has

$$
C_{Y \mid X}^{\infty}=\gamma^{-1} \gamma_{*} \mu \operatorname{hom}\left(\mathbb{C}_{Y}, \mathcal{O}_{X}\right)[d]=\gamma^{-1} \gamma_{*} C_{Y \mid X}^{\mathbb{R}},
$$

the holomorphic microfunctions along $Y$, where $\gamma$ is the projection of the cotangent bundle minus the zero section on the associated projectif bundle.

Similarly,

$$
C_{Y \mid X}=\gamma^{-1} \gamma_{*} t \mu \operatorname{hom}\left(\mathbb{C}_{Y}, \mathcal{O}_{X}\right)[d]=\gamma^{-1} \gamma_{*} C_{Y \mid X}^{\mathbb{R}, f}
$$

is the sheaf of microfunctions of finite order. The propagation in $C_{Y \mid X}^{\infty}$ was proved by Kashiwara-Schapira in [K-S2], and the propagation in $C_{Y \mid X}$ was studied by the author in [MF2], Schapira in [S] and Laurent in [L]. When we want to prove the propagation theorem for $t \mu \operatorname{hom}\left(F, \mathcal{O}_{X}\right)$, some of the essential tools developped in the preceding works are no longer available.

However, in the $\mathbb{C}$-constructible case we can use the theory of regular holonomic $\mathcal{D}$-modules, and in particular Kashiwara's theorem which asserts that $t \mathcal{H o m}\left(F, \mathcal{O}_{X}\right)$ has regular holonomic cohomology; moreover $\mu \operatorname{hom}\left(F, \mathcal{O}_{X}\right)$ and $t \mu \operatorname{hom}\left(F, \mathcal{O}_{X}\right)$ are obtained from $t \mathcal{H o m}\left(F, \mathcal{O}_{X}\right)$ tensorizing respectively by $\mathcal{E}_{X}^{\mathbb{R}}$, the sheaf of microlocal holomorphic operators, and by $\mathcal{E}_{X}^{\mathbb{R}, f}$, the subsheaf of tempered microlocal operators.

Using the identification of $X$ with the diagonal of $X \times X$, we can reduce the problem to the propagation of the solutions of $\mathcal{M}$ in $C_{Y \mid X}^{\mathbb{R}}$ and $C_{Y \mid X}^{\mathbb{R}, f}$ respectively, $Y$ an arbitrary complex submanifold, that is, $V=\dot{T}_{Y}^{*} X$, the conormal bundle to $Y$ minus the zero section.

The second essential tools are Bony's results concerning the propagation for solutions in the sheaf of tempered microfunctions $C^{f}$ for operators satisfying a Levi condition ([B]) together with a precised Cauchy-Kowalewski theorem of Kashiwara and Schapira for $C_{Y \mid X}^{\mathbb{R}}$ (cf. [K-S2]).

The paper is organized as follows : in the first section we construct the complex of sheaves of microlocal $F$-irregularity as the microlocalized of $I \mathcal{H o m}\left(F, \mathcal{O}_{X}\right)$ introduced in a previous work ([MF3]). Therefore, $I \mu$ hom $\left(F, \mathcal{O}_{X}\right)$ provides a natural third term to a distinguished triangle associated to the morphism

$$
t \mu \operatorname{hom}\left(F, \mathcal{O}_{X}\right) \rightarrow \mu \operatorname{hom}\left(F, \mathcal{O}_{X}\right)
$$


and represents the notion of $F$-irregularity : for example, when $F=\mathbb{C}_{Y}$ for a submanifold $Y$ of codimension $d, I \mu \operatorname{hom}\left(\mathbb{C}_{Y}[-d], \mathcal{O}_{X}\right)$ is nothing but the quotient $\frac{C_{Y \mid X}^{\mathbb{R}}}{C_{Y \mid X}^{\mathbb{R}, f}}$.

The second section is devoted to the propagation theorem in the $\mathbb{C}$-constructible perverse framework, and its successive reductions, for $t \mu$ hom $\left(F, \mathcal{O}_{X}\right)$, $\mu \operatorname{hom}\left(F, \mathcal{O}_{X}\right)$ and hence for $I \mu \operatorname{hom}\left(F, \mathcal{O}_{X}\right)$.

We are very happy to thank P. Schapira and M. Kashiwara for their useful suggestions, and V. Colin for her expertise on Andronikof's work.

\section{$\S 1$. Construction of $I \mu \operatorname{hom}\left(F, \mathcal{O}_{X}\right)$}

We shall recall some properties of the objects $t \mu \operatorname{hom}\left(F, \mathcal{O}_{X}\right)$ and $\mu \mathrm{hom}$ $\left(F, \mathcal{O}_{X}\right)$, where $X$ is an $n$-dimensional complex analytic manifold, $\mathcal{O}_{X}$ is the sheaf of holomorphic functions and $F$ is an object of $D_{\mathbb{R}-c}^{b}(X)$, that is a complex of sheaves of $\mathbb{C}$-vector spaces with bounded and $\mathbb{R}$-constructible cohomology. Recall that $D_{\mathbb{C}-c}^{b}(X)$ denotes the subcategory of $D_{\mathbb{R}-c}^{b}(X)$ whose objects are the $\mathbb{C}$-constructible complexes. The functor $\mu$ hom was introduced in the $80^{\text {ties }}$ by Kashiwara and Schapira ([K-S3]), and the tempered version $t \mu$ hom was introduced by Andronikof ([A]). We also recall some facts about the functor $t \mathcal{H}$ om due to Kashiwara $([\mathrm{K}])$ which can be recovered by the restriction of $t \mu$ hom to the base $X$ of the cotangent bundle $T^{*} X \underset{\pi}{\rightarrow} X$.

Let $\mathcal{D}_{X}$ (resp. $\left.\mathcal{D}_{X}^{\infty}\right)$ be the sheaf on $X$ of holomorphic differential operators of finite order (resp. infinite order), $\mathcal{E}_{X}$ (resp. $\mathcal{E}_{X}^{\infty}$ ) the sheaf of microdifferential operators of finite (resp. infinite) order, $\mathcal{E}_{X}^{\mathbb{R}, f}$ (resp. $\mathcal{E}_{X}^{\mathbb{R}}$ ) the sheaf of tempered microlocal operators (resp. microlocal operators).

$\mathcal{E}_{X}, \mathcal{E}_{X}^{\infty}$, as well as $\mathcal{E}_{X}^{\mathbb{R}, f}$ and $\mathcal{E}_{X}^{\mathbb{R}}$ are sheaves on $T^{*} X$, satisfying

$$
\begin{aligned}
\left.\mathcal{E}_{X}^{\mathbb{R}, f}\right|_{X} & =\left.\mathcal{E}_{X}\right|_{X}=\mathcal{D}_{X} \\
\left.\mathcal{E}_{X}^{\mathbb{R}}\right|_{X} & =\left.\mathcal{E}_{X}^{\infty}\right|_{X}=\mathcal{D}_{X}^{\infty}
\end{aligned}
$$

and they are all particular cases of $\mu$ hom and $t \mu$ hom. One denotes $\mathcal{E}_{X}(m)$ (resp. $\mathcal{D}_{X}(m)$ ) the sheaf of operators of order at most $m$.

For instance, when $F=\mathbb{C}_{Y}$, for a smooth complex submanifold of $X$ of codimension $d, \mu \operatorname{hom}\left(\mathbb{C}_{Y}, \mathcal{O}_{X}\right)$ is the sheaf $C_{Y \mid X}^{\mathbb{R}}[-d]$ of $[\mathrm{S}-\mathrm{K}-\mathrm{K}]$ and $t \mu \operatorname{hom}\left(\mathbb{C}_{Y}, \mathcal{O}_{X}\right)$ is the sheaf $C_{Y \mid X}^{\mathbb{R}, f}[-d]$ defined in $[\mathrm{A}]$.

When $F=\left(\mathbb{C}_{M}\right)^{\prime}$, the dual of $\mathbb{C}_{M}$, for a real analytic submanifold $M$ of $X$ such that $X$ is a complexified of $M, \mu \operatorname{hom}\left(\left(\mathbb{C}_{M}\right)^{\prime}, \mathcal{O}_{X}\right)[n]$ is isomorphic to the sheaf of microfunctions on $M$ and $t \mu \operatorname{hom}\left(\left(\mathbb{C}_{M}\right)^{\prime}, \mathcal{O}_{X}\right)[n]$ is isomorphic to the tempered microfunctions (cf. $[\mathrm{A}]$ and $[\mathrm{B}])$. 
When restricting to $X$ one obtains

$$
\left.\mu \operatorname{hom}\left(F, \mathcal{O}_{X}\right)\right|_{X} \simeq \mathbb{R} \mathcal{H o m}\left(F, \mathcal{O}_{X}\right),
$$

a complex of $\mathcal{D}_{X}^{\infty}$-modules, and

$$
\left.t \mu \operatorname{hom}\left(F, \mathcal{O}_{X}\right)\right|_{X} \simeq t \mathcal{H o m}\left(F, \mathcal{O}_{X}\right),
$$

a complex of $\mathcal{D}_{X}$-modules.

One also defines $I \mathcal{H}$ om $\left(F, \mathcal{O}_{X}\right)$, the sheaf of analytic local $F$-irregularity which appears to be a natural third term to a distinguished triangle

$$
t \mathcal{H o m}\left(F, \mathcal{O}_{X}\right) \rightarrow \mathbb{R} \mathcal{H o m}\left(F, \mathcal{O}_{X}\right) \rightarrow I \mathcal{H} \operatorname{om}\left(F, \mathcal{O}_{X}\right) \underset{+1}{\longrightarrow}
$$

where the left arrow is the usual one. In particular, when $F=\left(\mathbb{C}_{M}\right)^{\prime}$ one obtains

$$
I \mathcal{H o m}\left(F, \mathcal{O}_{X}\right)=\frac{B_{M}}{D b_{M}}
$$

where $B_{M}$ is the sheaf of hyperfunctions on $M$ and $D b_{M}$ is the sheaf of distributions on $M$; and when $F=\mathbb{C}_{Y}, Y$ a $d$-codimensional submanifold,

$$
I \mathcal{H o m}\left(\mathbb{C}_{Y}[-d], \mathcal{O}_{X}\right)=\frac{B_{Y \mid X}^{\infty}}{B_{Y \mid X}},
$$

where $B_{Y \mid X}^{\infty}$ (resp. $B_{Y \mid X}$ ) denotes the sheaf of holomorphic hyperfuntions (resp. holomorphic hyperfunctions with finite order) along $Y$.

Let us recall that $I \mathcal{H} \operatorname{om}\left(F, \mathcal{O}_{X}\right)$ has $\mathcal{D}_{X}$-module cohomology.

Let now $\Delta$ be the diagonal of $X \times X$ and $\tau: T_{\Delta}(X \times X) \rightarrow \Delta$ be the projection of the normal bundle to $\Delta$. Let $\tilde{X}$ be the complex normal deformation of $X \times X$ along $\Delta$ ( $\Delta$ identified with $X$ by the first projection $\left.p_{1}: X \times X \rightarrow X\right)$, let $p: \tilde{X} \rightarrow X \times X$ be the deformation morphism, let $t: \tilde{X} \rightarrow \mathbb{C}$ be the natural projection, let $\Omega=t^{-1}\left(\mathbb{R}^{+}\right)$, and let $\Omega \hookrightarrow{ }_{j} \tilde{X}$ and $T_{\Delta}(X \times X) \hookrightarrow_{s} \tilde{X}$ be the natural inclusions. (For more details we $\operatorname{refer}^{j}[\mathrm{~A}]$ (Prop. 3.2.1) and [K-S3].) Let $p_{2}$ denote the second projection of $X \times X$ on $X$. We have a distinguished triangle

$$
\begin{aligned}
t \mathcal{H o m}\left(\left(p^{!} p_{2}^{-1} F\right)_{\Omega}, \mathcal{O}_{\tilde{X}}\right) & \rightarrow \mathbb{R} \mathcal{H o m}\left(\left(p^{!} p_{2}^{-1} F\right)_{\Omega}, \mathcal{O}_{\tilde{X}}\right) \\
& \rightarrow \operatorname{IHom}\left(\left(p^{!} p_{2}^{-1} F\right)_{\Omega}, \mathcal{O}_{\tilde{X}}\right) \underset{+1}{\rightarrow} .
\end{aligned}
$$

Following the constructions in ([A], Lemme 2.1.8) we apply the functor

$$
s^{-1}\left(\mathcal{D}_{X \times X \leftarrow \tilde{X}} \underset{\mathcal{D}_{\tilde{X}}}{\mathbb{L}}\right) .
$$


to (1) and set

$$
\begin{aligned}
& \left.\operatorname{I\nu hom}\left(F, \mathcal{O}_{X}\right)\right):=
\end{aligned}
$$

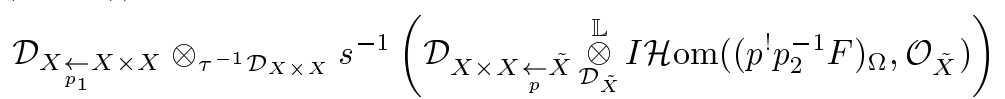

$$
\begin{aligned}
& \left.\nu \operatorname{hom}\left(F, \mathcal{O}_{X}\right)\right):=
\end{aligned}
$$

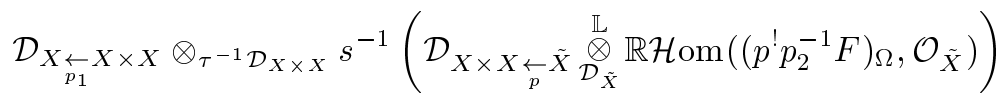

$$
\begin{aligned}
& \left.t \nu \operatorname{hom}\left(F, \mathcal{O}_{X}\right)\right):=
\end{aligned}
$$

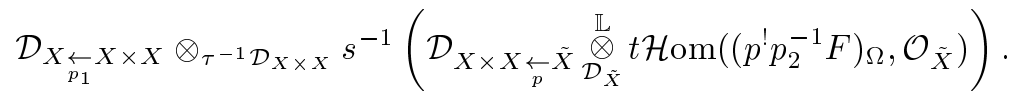

Then

$$
t \nu \operatorname{hom}\left(F, \mathcal{O}_{X}\right) \rightarrow \nu \operatorname{hom}\left(F, \mathcal{O}_{X}\right) \rightarrow I \nu \operatorname{hom}\left(F, \mathcal{O}_{X}\right) \underset{+1}{\rightarrow}
$$

is a distinguished triangle in $D^{b}\left(\tau^{-1} \mathcal{D}_{X}\right)$, the derived category whose objects are complexes of $\tau^{-1} \mathcal{D}_{X}$-modules with bounded cohomology. Finally, denoting by $\wedge$ the Fourier transform from $D_{\mathbb{R}^{+}}^{b}\left(T_{X}(X \times X)\right)$ to $D_{\mathbb{R}^{+}}^{b}\left(T_{X}^{*}(X \times X)\right)$, we define $I \mu \operatorname{hom}\left(F, \mathcal{O}_{X}\right)$ by

$$
I \mu \operatorname{hom}\left(F, \mathcal{O}_{X}\right):=\operatorname{I\nu hom}\left(F, \mathcal{O}_{X}\right)^{\wedge} .
$$

Here $D_{\mathbb{R}^{+}}^{b}(E)$, where $E$ is a real vector bundle on $X$, denotes the derived category of complexes of sheaves on $E$ of $\mathbb{C}$-vector spaces with bounded and $\mathbb{R}^{+}$-conic cohomology.

One easily deduces the isomorphisms

$$
\left.I \mu \operatorname{hom}\left(F, \mathcal{O}_{X}\right)\right|_{X} \simeq \mathbb{R} \pi_{*} I \mu \operatorname{hom}\left(F, \mathcal{O}_{X}\right) \simeq \operatorname{IHom}\left(F, \mathcal{O}_{X}\right)
$$

from the analogous formulae for $t \mu$ hom and $\mu$ hom. Moreover,

$$
\mathbb{R} \pi ! I \mu \operatorname{hom}\left(F, \mathcal{O}_{X}\right)=0
$$

by $(2.3 .2)$ of $[\mathrm{A}]$.

Also, as pointed out above, the correspondence

$$
F \rightarrow I \mu \operatorname{hom}\left(F, \mathcal{O}_{X}\right)
$$

defines a contravariant functor from $D_{\mathbb{R}-c}^{b}(X)$ to $D^{b}\left(\tau^{-1} \mathcal{D}_{X}\right)$.

On the other hand, since $I \mu \operatorname{hom}\left(F, \mathcal{O}_{X}\right)$ is the third term of a distinguished triangle where the other two terms are supported by the microsupport of $F, S S(F), I \mu \operatorname{hom}\left(F, \mathcal{O}_{X}\right)$ is also supported by $S S(F)$. 
Finally, we observe that the cohomology of $I \mu \operatorname{hom}\left(F, \mathcal{O}_{X}\right)$ is obviously provided of a canonical structure of $\mathcal{E}_{X}$-modules, induced by the structure on $t \mu \operatorname{hom}\left(F, \mathcal{O}_{X}\right)$ and $\mu \operatorname{hom}\left(F, \mathcal{O}_{X}\right)$ (cf. (TR4) of Proposition 1.4.4 of [K-S3]).

Definition 1.1. For any $F \in D_{\mathbb{R}-c}^{b}(X), I \mu \operatorname{hom}\left(F, \mathcal{O}_{X}\right)$ is the complex of microlocal analytic $F$-irregularity.

\section{$\S 2 . \quad$ Statement of the Main Theorem and Reductions}

In this section we start by briefly recalling the essential results on the 1-microcharacteristic variety of a coherent $\mathcal{E}_{X}$-module $\mathcal{M}$ along a smooth involutive manifold $V$ of $\dot{T}^{*} X$. Here $\dot{T}^{*} X$ denotes the complementary of the null section $X \hookrightarrow T^{*} X$ in $T^{*} X$. The 1-microcharacteristic variety $C_{V}^{1}(\mathcal{M})$ is a conic involutive closed analytic subset of $T_{V}\left(T^{*} X\right)$. To build it one needs to introduce the subring $\mathcal{E}_{V}$ of $\mathcal{E}_{X}$ generated by the operators of order at most one with a principal symbol vanishing on $V$; when $P \in \mathcal{E}_{V}(m):=\mathcal{E}_{X}(m) \cap \mathcal{E}_{V}$, modulo $\mathcal{E}_{V}(m-1)$, the symbol $\sigma_{V}^{1}(P)$ is a homogeneous function on $T_{V}\left(T^{*} X\right)$ and when $\mathcal{S}$ is a coherent ideal of $\mathcal{E}_{X}, C_{V}^{1}\left(\mathcal{E}_{X} / \mathcal{S}\right)$ is the subset of zeros of $\sigma_{V}^{1}\left(\mathcal{S} \cap \mathcal{E}_{V}\right)$. For further details see [MF1], [L] and [S].

Recall that if $\eta \in T_{V}\left(T^{*} X\right)$, one says that $\eta$ is non 1-microcharacteristic for $\mathcal{M}$ along $V$ if $\eta \notin C_{V}^{1}(\mathcal{M})$. Moreover, the normal cone $C_{V}(\mathrm{Car} \mathcal{M})$ is contained in $C_{V}^{1}(\mathcal{M})$. When $\mathcal{M}$ is a coherent $\mathcal{D}_{X^{-}}$or $\mathcal{E}_{X^{-}}$-module, $\operatorname{Car}(\mathcal{M})$ will denote its characteristic variety in $T^{*} X$. Let $F \in D_{\mathbb{R}-c}^{b}(X), S S(F)$ its microsupport in $T^{*} X$ (see [K-S3]); recall that $\operatorname{supp}(F)=S S(F) \cap X$. Moreover, if $\mathcal{M}$ is a coherent $\mathcal{D}_{X}$-module, $\operatorname{Car}(\mathcal{M})=S S\left(\mathbb{R} \mathcal{H} \operatorname{om}_{\mathcal{D}_{X}}\left(\mathcal{M}, \mathcal{O}_{X}\right)\right)$. Let us recall that $C_{V}(\operatorname{Car} \mathcal{M}):=C_{V}(\mathcal{M})$ was studied in [K-S1, K-S2]. It is called the microcharacteristic variety of $\mathcal{M}$ along $V$.

Let us denote by $D_{r h}^{b}\left(\mathcal{D}_{X}\right)$ the derived category whose objects are the complexes of left $\mathcal{D}_{X}$-modules with bounded regular holonomic cohomology. Recall that, as proved in $[\mathrm{K}]$, when $F \in D_{\mathbb{C}-c}^{b}(X)$, there exists a unique $\mathcal{N} \in$ $D_{r h}^{b}\left(\mathcal{D}_{X}\right)$ such that $\mathbb{R} \mathcal{H} \operatorname{om}_{\mathcal{D}_{X}}\left(\mathcal{N}, \mathcal{O}_{X}\right) \simeq F$ and that correspondence is an equivalence of categories. More precisely, $\mathcal{N}=t \mathcal{H o m}\left(F, \mathcal{O}_{X}\right)$ and by ([A], Theorem 4.2.6)

$$
\mathcal{E}_{X}^{\mathbb{R}, f} \underset{\pi^{-1} \mathcal{D}_{X}}{\otimes} \mathcal{N} \simeq t \mu \operatorname{hom}\left(F, \mathcal{O}_{X}\right)
$$

as well as

$$
\mathcal{E}_{X}^{\mathbb{R}} \underset{\pi^{-1} \mathcal{D}_{X}}{\otimes} \mathcal{N} \simeq \mu \operatorname{hom}\left(F, \mathcal{O}_{X}\right)
$$

(cf. $[\mathrm{K}-\mathrm{S} 4])$. 
Let $V$ be a smooth submanifold of a manifold $X$. One denotes $\rho_{V}$ the projection

$$
V \underset{X}{\times} T X \rightarrow T_{V} X
$$

In most cases studied in this paper, instead of $X$ we consider the cotangent bundle $T^{*} X$ and $V$ will be an involutive smooth submanifold; we then get

$$
\rho_{V}: \underset{T^{*} X}{\times T^{*}}\left(T^{*} X\right) \rightarrow T_{V}\left(T^{*} X\right)
$$

Here we identify $T\left(T^{*} X\right)$ and $T^{*}\left(T^{*} X\right)$ by $-H$, where $H$ is the Hamiltonian isomorphism. Recall that, if $(x ; \xi)$ denotes a system of local canonical coordinates on $T^{*} X$ in a neighborhood of $p \in T^{*} X$, if $(x, \xi ; \zeta, \eta)$ denotes the associated canonical coordinates on $T^{*}\left(T^{*} X\right),-H_{p}(\zeta d x+\eta d \xi)=(\zeta \partial / \partial \xi-\eta \partial / \partial x) \epsilon$ $T_{p}\left(T^{*} X\right)$

Let $\Omega_{X}$ denote the sheaf of holomorphic differential $n$-forms on $X$.

Theorem 2.1. Let $F \in D_{\mathbb{C}-c}^{b}(X)$ be perverse and $\mathcal{M}$ be a coherent $\mathcal{E}_{X}$ module. Let $V$ be smooth involutive in $\dot{T}^{*} X$ such that $S S(F) \subset V$. Then one has the inclusions

$$
\begin{array}{ll}
\text { a) } & \rho_{V}\left(S S\left(\mathbb{R H o m} \mathcal{E}_{X}\left(\mathcal{M}, \mu \operatorname{hom}\left(F, \mathcal{O}_{X}\right)\right)\right) \subset C_{V}(\mathcal{M}),\right. \\
\text { b) } & \rho_{V}\left(S S\left(\mathbb{R} \mathcal{H} \text { om }_{\mathcal{E}_{X}}\left(\mathcal{M}, t \mu \operatorname{hom}\left(F, \mathcal{O}_{X}\right)\right)\right) \subset C_{V}^{1}(\mathcal{M}),\right. \\
\text { c) } & \rho_{V}\left(S S\left(\mathbb{R} \mathcal{H} \text { om }_{\mathcal{E}_{X}}\left(\mathcal{M}, I \mu \operatorname{hom}\left(F, \mathcal{O}_{X}\right)\right)\right) \subset C_{V}^{1}(\mathcal{M}) .\right.
\end{array}
$$

Proof. c) It derives from a) and b).

a) and b) We may assume that $\mathcal{N}=t \mathcal{H o m}\left(F, \mathcal{O}_{X}\right)$ is concentrated in degree zero. Let us identify $T_{\Delta}^{*}(X \times X)$ with $T^{*} X$ and denote by $j: T^{*} X \hookrightarrow T^{*}(X \times X)$ the associated inclusion. Denote by $*$ the duality functor

$$
\mathbb{R} \mathcal{H} \operatorname{om}_{\mathcal{E}_{X}}\left(\cdot, \mathcal{E}_{X}\right) \underset{\pi^{-1} \mathcal{O}_{X}}{\otimes} \pi^{-1} \Omega_{X}^{\otimes-1}[n]
$$

Hence it is suffitient to prove that

$$
\rho_{V}\left(S S\left(j^{-1} \mathbb{R} \mathcal{H} \operatorname{om}_{\mathcal{E}_{X \times X}}\left(\mathcal{M} \underline{\underline{\otimes}}\left(\mathcal{E}_{X} \underset{\pi^{-1} \mathcal{D}_{X}}{\otimes} \pi^{-1} \mathcal{N}\right)^{*}, C_{\Delta \mid X \times X}^{\mathbb{R}}\right)\right)\right) \subset C_{V}(\mathcal{M}),
$$

as well as with $C_{\Delta \mid X \times X}^{\mathbb{R}}$ replaced by $C_{\Delta \mid X \times X}^{\mathbb{R}, f}$, and $C_{V}(\mathcal{M})$ replaced by $C_{V}^{1}(\mathcal{M})$. Since $C_{\Delta \mid X \times X}^{\mathbb{R}}$ is supported by $T_{\Delta}^{*}(X \times X)$,

$$
\begin{aligned}
& S S\left(j^{-1} \mathbb{R} \mathcal{H o m} \mathcal{E}_{X \times X}\left(\mathcal{M} \underline{\underline{\underline{N}}} \tilde{\mathcal{N}}^{*}, C_{\Delta \mid X \times X}^{\mathbb{R}}\right)\right) \\
& \subset \rho_{T_{\Delta}^{*}(X \times X)}\left(S S\left(\mathbb{R} \mathcal{H} \operatorname{om}_{\mathcal{E}_{X \times X}}\left(\mathcal{M} \underline{\boldsymbol{\nabla}} \tilde{\mathcal{N}}^{*}, C_{\Delta \mid X \times X}^{\mathbb{R}}\right)\right)\right)
\end{aligned}
$$


where $\tilde{\mathcal{N}}=\mathcal{E}_{X} \underset{\pi^{-1} \mathcal{D}_{X}}{\otimes} \pi^{-1} \mathcal{N}$, and the inclusion holds for $C_{\Delta \mid X \times X}^{\mathbb{R}, f}$ as well. Here we identify $T_{T_{\Delta}^{*}(X \times X)}\left(T^{*}(X \times X)\right)$ with $T^{*}\left(T^{*} X\right)$ via the identification of $T_{T_{\Delta}^{*}(X \times X)}\left(T^{*}(X \times X)\right)$ with $T^{*}\left(T_{\Delta}^{*}(X \times X)\right)$ and the identification of the last one with $T^{*}\left(T^{*} X\right)$.

Suppose we know that for any $\mathcal{E}_{X}$-coherent module, for any smooth submanifold $Y$ of $X$,

$$
\begin{aligned}
& \rho_{\dot{T}_{Y}^{*} X}\left(S S\left(\mathbb{R} \mathcal{H} \operatorname{om}_{\mathcal{E}_{X}}\left(\mathcal{M}, C_{Y \mid X}^{\mathbb{R}}\right)\right)\right) \subset C_{\dot{T}_{Y}^{*} X}(\mathcal{M}), \\
& \rho_{{\dot{T_{Y}^{*}}}^{*} X}\left(S S\left(\mathbb{R} \mathcal{H} \operatorname{om}_{\mathcal{E}_{X}}\left(\mathcal{M}, C_{Y \mid X}^{\mathbb{R}, f}\right)\right)\right) \subset C_{\dot{T}_{Y}^{*} X}^{1}(\mathcal{M}),
\end{aligned}
$$

where $\rho_{\dot{T}_{Y}^{*} X}$ is, as before, the projection

$$
\dot{T}_{Y}^{*} X \underset{T^{*} X}{\times} T^{*}\left(T^{*} X\right) \rightarrow T_{\dot{T}_{Y}^{*} X}\left(T^{*} X\right) .
$$

Then, replacing $\mathcal{M}$ by $\mathcal{M} \unrhd \tilde{\mathcal{N}}^{*}$ we get

$$
\begin{aligned}
& \rho_{\dot{T}_{\Delta}^{*}(X \times X)}\left(S S \left(\mathbb{R} \mathcal{H} \operatorname{mom}_{\mathcal{E}_{X \times X}}\right.\right.\left.\left.\left(\mathcal{M} \underline{\underline{\nabla}} \tilde{\mathcal{N}}^{*}, C_{\Delta \mid X \times X}^{\mathbb{R}}\right)\right)\right) \\
& \subset C_{\dot{T}_{\Delta}^{*}(X \times X)}\left(\mathcal{M} \underline{\otimes} \tilde{\mathcal{N}}^{*}\right):=C(\mathcal{M}, \mathcal{N}) \\
& \rho_{\dot{T}_{\Delta}^{*}(X \times X)}\left(S S\left(\mathbb{R} \mathcal{H} \operatorname{om}_{\mathcal{E}_{X \times X}}\left(\mathcal{M} \underline{\tilde{\mathcal{N}}^{*}}, C_{\Delta \mid X \times X}^{\mathbb{R}, f}\right)\right)\right) \\
& \subset C_{\dot{T}_{\Delta}^{*}(X \times X)}^{1}\left(\mathcal{M} \underline{\underline{\nabla}} \tilde{\mathcal{N}}^{*}\right):=C^{1}(\mathcal{M}, \mathcal{N})
\end{aligned}
$$

where we identify $T^{*}(X \times X)$ to $T^{*} X$ by the first projection.

Since $S S(F)$ equals the characteristic variety of $\mathcal{N}$, we know by [K-O] that $\mathcal{N}$, being regular holonomic, is regular along $V$. We shall use the following result, which is a slight improvement of the analogous in [MF1].

Lemma 2.2. Let $X$ be a complex analytic manifold. Let $\mathcal{N}$ be a coherent $\mathcal{E}_{X}$-module regular along a smooth involutive submanifold $V \subset \dot{T}^{*} X$. Then, for any coherent $\mathcal{E}_{X}$-module $\mathcal{M}$,

$$
\begin{aligned}
\rho_{V}(C(\mathcal{M}, \mathcal{N})) & \subset C_{V}(\mathcal{M}), \\
\rho_{V}\left(C^{1}(\mathcal{M}, \mathcal{N})\right) & \subset C_{V}^{1}(\mathcal{M}) .
\end{aligned}
$$

Proof. The first inclusion is obvious since $C(\mathcal{M}, \mathcal{N})=C(\operatorname{Car}(\mathcal{M})$, $\operatorname{Car}(\mathcal{N}))$ and $\operatorname{Car}(\mathcal{N}) \subset V$.

As for the second inclusion, let us start by assuming that $V$ is regular involutive, that is, the canonical 1-form never vanishes on $V$. By [K-O], we 
know that $\mathcal{N}$ is locally a quotient of some power $N$ of a coherent $\mathcal{E}_{X}$-module $\mathcal{L}_{V}$ supported by $V$, with simple characteristics. Hence

$$
C^{1}(\mathcal{M}, \mathcal{N}) \subset C^{1}\left(\mathcal{M}, \mathcal{L}_{V}^{N}\right)
$$

and then we apply Theorem 1.4.2 in [MF1].

For the general case, we use the dummy variable trick, that is, we consider local canonical coordinates $(x, \xi)$ in $T^{*} X$ in a neighborhood of $V$, the regular involutive submanifold of $\dot{T}^{*}(X \times \mathbb{C})$,

$$
\tilde{V}=\left\{(x, t ; \xi, \zeta) \in \dot{T}^{*} X \times \dot{T}^{*} \mathbb{C} ;(x ; \xi) \in V, \zeta \neq 0\right\}
$$

and $\tilde{\mathcal{M}}:=\mathcal{M} \underline{\underline{\otimes}} \mathcal{E}_{\mathbb{C}}$. Since

$$
C_{\tilde{V}}^{1}(\tilde{\mathcal{M}})=C_{V}^{1}(\mathcal{M}) \times \dot{T}^{*} \mathbb{C},
$$

we get

$$
\rho_{\tilde{V}}\left(C^{1}(\tilde{\mathcal{M}}, \tilde{\mathcal{N}})\right) \subset C_{\tilde{V}}^{1}(\tilde{\mathcal{M}})=C_{V}^{1}(\mathcal{M}) \times \dot{T}^{*} \mathbb{C} .
$$

On the other hand,

$$
\rho_{\tilde{V}}\left(C^{1}(\tilde{\mathcal{M}}, \tilde{\mathcal{N}})\right)=\rho_{V}\left(C^{1}(\mathcal{M}, \mathcal{N})\right) \times \dot{T}^{*} \mathbb{C}
$$

hence the result.

Therefore, a) and b) of Theorem 2.1 hold provided that we prove (4).

Let $f: \tilde{X} \rightarrow X$ be a smooth morphism of complex manifolds, let $\Lambda$ be a smooth involutive submanifold of $\dot{T}^{*} X$, let $\omega: \tilde{X} \underset{X}{\times} T^{*} X \rightarrow T^{*} X$ and ${ }^{t} f^{\prime}: \tilde{X} \underset{X}{\times}$ $T^{*} X \rightarrow T^{*} \tilde{X}$ be the canonical morphisms. Let $W:={ }^{t} f^{\prime} \circ \omega^{-1}(\Lambda)$. Then $W$ is a smooth involutive submanifold of $\dot{T}^{*} \tilde{X}$. In this situation, we have:

Lemma 2.3. Let $f: \tilde{X} \rightarrow X$ be a smooth analytic morphism of finite dimensional complex analytic manifolds. Let $\Lambda$ be a smooth involutive submanifold of $\dot{T}^{*} X$ and let $W={ }^{t} f^{\prime} \circ \omega^{-1}(\Lambda) \subset \dot{T}^{*} \tilde{X}$. Set $\omega^{N}: T_{\omega^{-1}(\Lambda)}\left(T^{*} X \underset{X}{\times} \tilde{X}\right) \rightarrow$ $T_{\Lambda}\left(T^{*} X\right)$ and $\pi^{N}: T_{\omega^{-1}(\Lambda)}\left(T^{*} X \underset{X}{\times} \tilde{X}\right) \rightarrow T_{W}\left(T^{*} \tilde{X}\right)$, the canonical morphisms associated to $\omega$ and ${ }^{t} f^{\prime}$.

Then:

i) $\pi^{N}$ is injective.

ii) Let $\mathcal{M}$ be a coherent $\mathcal{E}_{X}$-module. We have the following estimation:

$$
C_{W}^{1}\left(\underline{f}^{*} \mathcal{M}\right)=\pi^{N}\left(\omega^{N}\right)^{-1} C_{\Lambda}^{1}(\mathcal{M}) .
$$


Proof. Since the statement is of local nature, we may assume that $\tilde{X} \simeq$ $X \times Y$, that $f: \tilde{X} \times Y \rightarrow X$ is the projection, and consider local coordinates on $X \times Y,\left(x, x^{\prime}\right)$, such that $x$ are local coordinates on $X, x^{\prime}$ are local coordinates on $Y$ and $f\left(x, x^{\prime}\right)=x$. Consider the associated canonical coordinates $\left(x, x^{\prime} ; \xi, \xi^{\prime}\right)$ on $T^{*} \tilde{X}$. We get

$$
\begin{gathered}
\tilde{X} \underset{X}{\times} T^{*} X=Y \times T^{*} X, \\
W=\left\{\left(x^{\prime}, \xi^{\prime}\right) \in T^{*} Y, \xi^{\prime}=0\right\} \times \Lambda=Y \times \Lambda, \\
T_{W}\left(T^{*} \tilde{X}\right) \simeq T_{Y}\left(T^{*} Y\right) \times T_{\Lambda}\left(T^{*} X\right), \\
T_{\omega^{-1}(\Lambda)}\left(\underset{X}{\tilde{X}} \underset{X}{\times} T^{*} X\right) \simeq Y \times T_{\Lambda}\left(T^{*} X\right),
\end{gathered}
$$

and, for $x^{\prime} \in Y$ and $p \in T_{\Lambda}\left(T^{*} X\right), \pi^{N}\left(x^{\prime}, p\right)=\left(\left(x^{\prime} ; 0\right), p\right)$. Here we identify $Y$ to the zero sections of $T Y$, of $T^{*} Y$ and of $T_{Y}\left(T^{*} Y\right)$. This proves i).

As for ii), it will be enough to consider the case where $\mathcal{M}$ is of the form $\mathcal{M}=\frac{\mathcal{E}_{X}}{\mathcal{J}}$ with $\mathcal{J}$ a coherent ideal of $\mathcal{E}_{X}$. In that case, $\underline{f}^{*} \mathcal{M}$ is isomorphic to $\frac{\mathcal{E}_{\tilde{X}}}{\mathcal{E}_{\tilde{X}} \mathcal{J}+\mathcal{E}_{\tilde{X}} D_{x^{\prime}}}$, where $\mathcal{E}_{\tilde{X}} D_{x^{\prime}}$ denotes the ideal generated by the derivations in the $x^{\prime}$ variables. Hence,

$$
C_{W}^{1}\left(\underline{f}^{*} \mathcal{M}\right)=\left\{(q, p) \in T_{Y}\left(T^{*} Y\right) \times T_{\Lambda}\left(T^{*} X\right), q \in Y, p \in C_{\Lambda}^{1}(\mathcal{M})\right\},
$$

hence the result.

We shall now return to (4).

Lemma 2.4. Let $Y$ be a smooth submanifold of $X$. Then, for any coherent $\mathcal{E}_{X}$-module $\mathcal{M}$,

$$
\begin{aligned}
& \left.\mathrm{a}^{\prime}\right) \quad \rho_{\dot{T}_{Y}^{*} X}\left(S S\left(\mathbb{R} \mathcal{H} \operatorname{com}_{\mathcal{E}_{X}}\left(\mathcal{M}, C_{Y \mid X}^{\mathbb{R}}\right)\right)\right) \subset C_{\dot{T}_{Y}^{*} X}(\mathcal{M}) \text {, } \\
& \left.\mathrm{b}^{\prime}\right) \quad \rho_{\dot{T}_{Y}^{*} X}\left(S S\left(\mathbb{R} \mathcal{H} \operatorname{com}_{\mathcal{E}_{X}}\left(\mathcal{M}, C_{Y \mid X}^{\mathbb{R}, f}\right)\right)\right) \subset C_{\dot{T}_{Y}^{*} X}^{1}(\mathcal{M}) \text {. }
\end{aligned}
$$

Proof. a') It is a form of Theorem 8.2.1 of [K-S1]. This proves a) of Theorem 2.1.

b') Since the statements are local and invariant by canonical transformation, we may assume that $Y$ is a hypersurface.

Let $(x, t)=\left(x_{1}, \cdots, x_{n-1}, t\right)$ be local coordinates on $X$ such that $t=0$ defines $Y,(x, t ; \xi, \zeta)$ the associated canonical coordinates in $T^{*} X$ and $\gamma$ be the section

$$
Y \underset{\gamma}{\hookrightarrow} \dot{T}_{Y}^{*} X, \quad \gamma(y)=(y ; 1)
$$


in the neighborhood of $0 \in Y$.

We shall regard $T^{*} Y\left(\simeq T^{*}(\gamma(Y))\right)$ as a submanifold of $T^{*}\left(\dot{T}_{Y}^{*} X\right)$ via the composition $\ell_{Y}$ of the morphisms:

$$
T^{*} Y \hookrightarrow T_{Y}^{*} X \underset{Y}{\times} T^{*} Y \hookrightarrow T^{*}\left(\dot{T}_{Y}^{*} X\right)
$$

where the left arrow derives from the section $\gamma$ and the right arrow is the immersion associated to $\dot{T}_{Y}^{*} X \rightarrow Y$.

More precisely, if $(x ; \xi) \in T^{*} Y, \ell_{Y}(x ; \xi)=(x, 1 ; \xi, 0) \in T^{*}\left(\dot{T}_{Y}^{*} X\right)$. Moreover, if $H$ denotes the Hamiltonian isomorphism, $-H$ induces an isomorphism:

$$
T^{*}\left(\dot{T}_{Y}^{*} X\right) \simeq T_{\dot{T}_{Y}^{*} X}\left(T^{*} X\right)
$$

(see (6.2.2) and (6.2.3) of [K-S 3]). We still denote by $-H$ this isomorphism for the sake of simplicity. Explicitely,

$$
-H \circ \ell_{Y}(x ; \xi)=-H(x, 1 ; \xi, 0)=(-\xi, 1 ; x, 0) .
$$

Therefore we may regard $T^{*} Y$ as a submanifold of $T_{\dot{T}_{Y}^{*} X}\left(T^{*} X\right)$ by the immersion $\tilde{\rho}_{Y}:=-H \circ \ell_{Y}$.

Let $V=\left\{\xi_{1}=\cdots=\xi_{n-1}=0 ; \zeta \neq 0\right\}$. The composition of the natural morphism of vector bundles $s_{Y}: T_{\dot{T}_{Y}^{*} X}\left(T^{*} X\right) \rightarrow T_{V}\left(T^{*} X\right)$ with $\tilde{\rho}_{Y}$ is injective ; more precisely, if $(x ; \xi) \in T^{*} Y$ then

$$
s_{Y} \tilde{\rho}_{Y}(x ; \xi)=(-\xi, 0,1 ; x) \in T_{V}\left(T^{*} X\right) .
$$

We set $\phi_{Y}:=s_{Y} \tilde{\rho}_{Y}$. By means of $\phi_{Y}$ we identify $T^{*} Y$ to a submanifold of $T_{V}\left(T^{*} X\right)$. Moreover, if $\mathcal{M}$ is an arbitrary $\mathcal{E}_{X^{-}}$coherent module we have an inclusion

$$
C_{V}^{1}(\mathcal{M}) \cap T^{*} Y \subset s_{Y}\left(C_{\dot{T}_{Y}^{*} X}^{1}(\mathcal{M}) \cap T^{*} Y\right)
$$

since the sheaf $\mathcal{E}_{V}$ is a subsheaf of $\mathcal{E}_{\dot{T}_{Y}^{*} X}$ and, by the above identification, for any $P \in \mathcal{E}_{V},\left.\sigma_{V}^{1}(P)\right|_{T^{*} Y}=\left.\sigma_{\dot{T}_{Y}^{*} X}^{1}(P)\right|_{T^{*} Y}$.

For any coherent $\mathcal{E}_{X}$-module $\mathcal{M}$ we regard $\mathcal{L}:=\mathbb{R} \mathcal{H} \operatorname{om}_{\mathcal{E}_{X}}\left(\mathcal{M}, C_{Y \mid X}^{\mathbb{R}, f}\right)$ as a complex on $\dot{T}_{Y}^{*} X$. Then, to get b'), it is enough to prove the inclusion

$$
\text { b") }-H(S S(\mathcal{L})) \subset C_{\dot{T}_{Y}^{*} X}^{1}(\mathcal{M}) .
$$

Assume that, for any coherent $\mathcal{E}_{X}$-module $\mathcal{M}$, the following inclusion holds :

$$
S S\left(\left.\mathcal{L}\right|_{\gamma(Y)}\right) \subset C_{V}^{1}(\mathcal{M}) \cap T^{*} Y
$$


Then, to get b"), we may use (8) by adjunction of a new variable following a suggestion of M. Kashiwara. Let $\tilde{X}=X \times \mathbb{C}$ with the coordinates $\left(x, t^{\prime}, s\right)$ and let $f: \tilde{X} \rightarrow X$ be the smooth morphism

$$
f\left(x, t^{\prime}, s\right)=\left(x, s e^{t^{\prime}}\right) .
$$

Let $\tilde{\mathcal{M}}=\underline{f}^{*} \mathcal{M}$ be the inverse image of $\mathcal{M}$, a coherent $\mathcal{E}_{\tilde{X}}$-module. Let $Y^{\prime} \subset \tilde{X}$ be defined by $s=0$ and let

$$
V^{\prime}=\left\{\left(x, t^{\prime}, s ; \xi, \zeta^{\prime}, \eta\right) ; \quad \xi=0, \quad \zeta^{\prime}=0, \quad \eta \neq 0\right\} \subset \dot{T}^{*} \tilde{X} .
$$

Let $f^{\prime}: T \tilde{X} \rightarrow T \underset{X}{\times} \tilde{X},{ }^{t} f^{\prime}: T^{*} X \underset{X}{\times} \tilde{X} \rightarrow T^{*} \tilde{X}$ and $\omega: T^{*} X \underset{X}{\times} \tilde{X} \rightarrow T^{*} X$ be the canonical morphisms:

$$
\dot{T}^{*} X \underset{\omega}{\leftarrow} \dot{T}^{*} X \underset{X}{\times} \tilde{X} \underset{{ }_{f^{\prime}}}{\rightarrow} \dot{T}^{*} \tilde{X}
$$

Explicitely

$$
f_{\left(x, t^{\prime}, s\right)}^{\prime}\left(\xi^{\prime}, \zeta^{\prime}, \eta\right)=\left(\xi^{\prime}, \zeta^{\prime} s e^{t^{\prime}}+\eta e^{t^{\prime}}\right)
$$

and

$$
{ }^{t} f_{\left(x, t^{\prime}, s\right)}^{\prime}(\xi, \zeta)=\left(\xi, \zeta s e^{t^{\prime}}, \zeta e^{t^{\prime}}\right) .
$$

Restricting to $\eta=1$ and fixing a determination of $\log \left(\frac{1}{\zeta}\right)$ we get a section $h$ of $\omega$

$$
h: \dot{T}^{*} X \rightarrow \dot{T}^{*} X \underset{X}{\times} \tilde{X}
$$

and $\bar{h}:={ }^{t} f^{\prime} \circ h$ gives an immersion of the corresponding open subdomain of $\left\{(x, t ; \xi, \zeta) \in T^{*} X ; \zeta \neq 0\right\}$ in $\left\{\left(x, t^{\prime}, s ; \xi^{\prime}, \zeta^{\prime}, \eta\right) \in T^{*} \tilde{X} ; \eta=1\right\}$. The image $\bar{h}\left(\dot{T}_{Y}^{*} X\right)$ is an open subset of $\dot{T}_{Y^{\prime}}^{*} \tilde{X} \cap\{\eta=1\}$. More precisely,

$$
h(x, t ; \xi, \zeta)=((x, t ; \xi, \zeta), \zeta t, \log (1 / \zeta))
$$

and

$$
\bar{h}(x, t ; \xi, \zeta)=(x, \log (1 / \zeta), \zeta t ; \xi, \zeta t, 1) .
$$

Remark that we can cover $\left\{p \in \dot{T}^{*} X ; \zeta \neq 0\right\}$ by two such open domains, $\Omega_{1}$ and $\Omega_{2}$. Since the notion of microsupport is of local nature, it is enough to prove b") for the restriction of $F$ to each of $\Omega_{1} \cap \dot{T}_{Y}^{*} X, \Omega_{2} \cap \dot{T}_{Y}^{*} X$. In that situation, $\bar{h}: \dot{T}^{*} X \rightarrow \dot{T}^{*} \tilde{X}$ induces an analytic isomorphism

$$
h_{Y}: \dot{T}_{Y}^{*} X \simeq \dot{T}_{\tilde{Y}}^{*}, \tilde{X} \cap\{\eta=1\} .
$$

More precisely, setting $i_{Y}: \dot{T}_{Y}^{*} X \hookrightarrow \dot{T}^{*} X$ and $i_{Y^{\prime}}^{\prime}: \dot{T}_{\tilde{Y}^{\prime}}^{*} \tilde{X} \cap\{\eta=1\} \hookrightarrow \dot{T}^{*} \tilde{X}$, we have

$$
\bar{h} \circ i_{Y}=i_{Y^{\prime}}^{\prime} \circ h_{Y} .
$$


Let $h^{c}$ be the canonical isomorphism

$$
T^{*}\left(\dot{T}_{Y}^{*} X\right) \rightarrow T^{*}\left(T_{Y^{\prime}}^{*} \tilde{X} \cap\{\eta=1\}\right)\left(\simeq T^{*} Y^{\prime}\right)
$$

induced by $h_{Y}$. Composing with the immersion

$$
\phi_{Y^{\prime}}: T^{*} Y^{\prime} \hookrightarrow T_{V^{\prime}}\left(T^{*} \tilde{X}\right)
$$

we get an isomorphism

$$
\left.T^{*}\left(T_{Y}^{*} X\right) \simeq T_{V^{\prime}}\left(T^{*} \tilde{X}\right)\right|_{\{s=0, \eta=1\}}
$$

We set

$$
\bar{h}^{N}:=\phi_{Y^{\prime}} \circ h^{c} \circ(-H)^{-1}: T_{\dot{T}_{Y}^{*} X}\left(T^{*} X\right) \rightarrow T_{V^{\prime}}\left(T^{*} \tilde{X}\right) .
$$

Remark that, by functoriality, $\bar{h}^{N}$ is also the quotient morphism associated to $\bar{h}$ and $\bar{h} \circ i_{Y}$. On the other hand, the sequence of morphisms of vector bundles :

$$
\dot{T}^{*} X \underset{h}{\rightarrow} \dot{T}^{*} X \underset{X}{\times} \underset{{ }_{t f^{\prime}}}{\rightarrow} \dot{T}^{*} \tilde{X}
$$

induces a sequence

$$
\dot{T}_{Y}^{*} X \underset{e^{\prime}}{\rightarrow} \dot{T}_{Y}^{*} X \underset{X}{\times} \tilde{X} \underset{\pi^{\prime}}{\rightarrow} V^{\prime}
$$

and $\pi^{\prime}$ is injective since ${ }^{t} f^{\prime}$ is injective. Setting $j_{Y^{\prime}}: \dot{T}_{Y^{\prime}}^{*} \tilde{X} \cap\{\eta=1\} \hookrightarrow V^{\prime}$, by construction

$$
j_{Y^{\prime}} \circ h_{Y}=\pi^{\prime} \circ e^{\prime}
$$

Set $\tilde{V}^{\prime}:=\dot{T}_{Y}^{*} X \underset{X}{\times} \tilde{X}$. Then

$$
\begin{gathered}
\tilde{V}^{\prime}=T^{*} \underset{X}{\times} \tilde{X} \cap \omega^{-1}\left(\dot{T}_{Y}^{*} X\right) \\
\pi^{\prime}\left(\tilde{V}^{\prime}\right)=\dot{T}_{Y^{\prime}}^{*} \tilde{X} \\
\pi^{\prime} e^{\prime}\left(\dot{T}_{Y}^{*} X\right)=h_{Y}\left(\dot{T}_{Y}^{*} X\right)=\dot{T}_{Y^{\prime}}^{*} \tilde{X} \cap\{\eta=1\} .
\end{gathered}
$$

Let

$$
\begin{gathered}
e^{N}: T_{\dot{T}_{Y}^{*} X} T^{*} X \rightarrow T_{\tilde{V}^{\prime}}\left(T^{*} X \underset{X}{\times} \tilde{X}\right), \\
\omega^{N}: T_{\tilde{V}^{\prime}}\left(T^{*} X \underset{X}{\underset{X}{X}}\right) \rightarrow T_{\dot{T}_{Y}^{*} X} T^{*} X, \\
\pi^{\prime N}: T_{\tilde{V}^{\prime}}\left(T^{*} X \underset{X}{\times} \tilde{X}\right) \rightarrow T_{V^{\prime}}\left(T^{*} \tilde{X}\right),
\end{gathered}
$$


be the canonical morphisms respectively associated to $h$ and $e^{\prime}$, to $\omega$ and $\left.\omega\right|_{\tilde{V}^{\prime}}$, to ${ }^{t} f^{\prime}$ and $\pi^{\prime}$. Consider the diagram of morphisms below:

$$
T^{*}\left(\dot{T}_{Y}^{*} X\right) \underset{-H}{\simeq} T_{\dot{T}_{Y}^{*} X} T^{*} X \underset{e^{N}}{\rightarrow} T_{\tilde{V}^{\prime}}\left(T^{*} X \underset{X}{\times} \tilde{X}\right) \underset{\pi^{\prime N}}{\rightarrow} T_{V^{\prime}}\left(T^{*} \tilde{X}\right) .
$$

(Here $-H$ denotes the isomorphism induced by the Hamiltonian isomorphism as before.) Remark that $\pi^{\prime N}$ is the composition of the morphisms:

$$
T_{\tilde{V}^{\prime}}\left(T^{*} \underset{X}{\underset{X}{\times}} \tilde{X}\right) \underset{\pi^{N}}{\rightarrow} T_{\dot{T}_{Y^{\prime}}^{*}, \tilde{X}^{\prime}} T^{*} \tilde{X} \underset{s_{Y^{\prime}}}{\rightarrow} T_{V^{\prime}}\left(T^{*} \tilde{X}\right) .
$$

Furthermore, $\left.s_{Y^{\prime}}\right|_{\dot{T}^{*} Y^{\prime}}$ is injective, $\pi^{N}$ is injective (cf. Lemma 2.3) and $\pi^{\prime N}$ 。 $e^{N}=\bar{h}^{N}=\phi_{Y^{\prime}} \circ h^{c} \circ(-H)^{-1}$. Since $h$ is a section of $\omega$, that is, $\omega \circ h=\mathrm{Id}$, by functoriality, $e^{N}$ is a section of $\omega^{N}$.

Recall we assumed (8), hence we have the inclusion

$$
S S\left(\left.\tilde{\mathcal{L}}\right|_{\{\eta=1\}}\right) \subset C_{V^{\prime}}^{1}(\tilde{\mathcal{M}}) \cap T^{*} Y^{\prime}
$$

where $\tilde{\mathcal{L}}$ denotes

$$
\mathbb{R}_{\mathcal{H}} \operatorname{om}_{\mathcal{E}_{\tilde{X}}}\left(\tilde{\mathcal{M}}, C_{Y^{\prime} \mid \tilde{X}}^{\mathbb{R}, f}\right) .
$$

On the other hand, for any coherent $\mathcal{E}_{X}$-module $\mathcal{M}$,

$$
h_{Y}^{-1}\left(\left.\mathbb{R} \mathcal{H} \operatorname{om}_{\mathcal{E}_{\tilde{X}}}\left(\tilde{\mathcal{M}}, C_{Y^{\prime} \mid \tilde{X}}^{\mathbb{R}, f}\right)\right|_{\eta=1}\right) \simeq \mathbb{R} \mathcal{H} \operatorname{om}_{\mathcal{E}_{X}}\left(\mathcal{M}, C_{Y \mid X}^{\mathbb{R}, f}\right)=\mathcal{L}
$$

To prove this, it is enough to consider $\mathcal{M} \simeq \mathcal{E}_{X}$, hence

$$
\tilde{\mathcal{M}}=\frac{\mathcal{E}_{\tilde{X}}}{\mathcal{E}_{\tilde{X}}\left(s D_{s}-D_{t^{\prime}}\right)}
$$

in which case the isomorphism above is clear.

Therefore,

$$
\begin{aligned}
S S(\mathcal{L}) & =\left(h^{c}\right)^{-1}\left(S S\left(\left.\tilde{\mathcal{L}}\right|_{\{\eta=1\}}\right)\right) \\
& \subset\left(h^{c}\right)^{-1}\left(C_{V^{\prime}}^{1}(\tilde{\mathcal{M}}) \cap T^{*} Y^{\prime}\right) \\
& \subset\left(h^{c}\right)^{-1}\left(s_{Y^{\prime}}\left(C_{\dot{T}_{Y^{\prime}}^{*} \tilde{X}}^{1}(\tilde{\mathcal{M}}) \cap T^{*} Y^{\prime}\right)\right) .
\end{aligned}
$$

In order to get b") we shall prove the inclusion

$$
-H\left(h^{c}\right)^{-1}\left(s_{Y^{\prime}}\left(C_{\dot{T}_{Y^{\prime}}^{*}, \tilde{X}}^{1}(\tilde{\mathcal{M}}) \cap T^{*} Y^{\prime}\right)\right) \subset C_{\dot{T}_{Y}^{*} X}^{1}(\mathcal{M}) .
$$


Using Lemma 2.3 with $\Lambda=T_{Y}^{*} X$, we have

$$
\begin{aligned}
-H\left(h^{c}\right)^{-1} & \left(s_{Y^{\prime}}\left(C_{\dot{T}_{Y^{\prime}}^{*} \tilde{X}}^{1}(\tilde{\mathcal{M}}) \cap T^{*} Y^{\prime}\right)\right) \\
= & -H\left(h^{c}\right)^{-1} \phi_{Y^{\prime}}^{-1}\left(s_{Y^{\prime}}\left(\pi^{N}\left(\omega^{N}\right)^{-1}\left(C_{\dot{T}_{Y}^{*} X}^{1}(\mathcal{M})\right) \cap T^{*} Y^{\prime}\right)\right) \\
& =\left(\bar{h}^{N}\right)^{-1}\left(s_{Y^{\prime}}\left(\pi^{N}\left(\omega^{N}\right)^{-1}\left(C_{\dot{T}_{Y}^{*} X}^{1}(\mathcal{M})\right) \cap T^{*} Y^{\prime}\right)\right) \\
& =\left(e^{N}\right)^{-1}\left(\pi^{\prime}\right)^{-1}\left(s_{Y^{\prime}}\left(\pi^{N}\left(\omega^{N}\right)^{-1}\left(C_{\dot{T}_{Y}^{*} X}^{1}(\mathcal{M})\right) \cap T^{*} Y^{\prime}\right)\right) \\
& =\left(e^{N}\right)^{-1}\left(\pi^{N}\right)^{-1} s_{Y^{\prime}}^{-1}\left(s_{Y^{\prime}}\left(\pi^{N}\left(\omega^{N}\right)^{-1}\left(C_{\dot{T}_{Y}^{*} X}^{1}(\mathcal{M})\right) \cap T^{*} Y^{\prime}\right)\right) \\
& \subset\left(e^{N}\right)^{-1}\left(\omega^{N}\right)^{-1}\left(C_{\dot{T}_{Y}^{*} X}^{1}(\mathcal{M})\right) \\
& \subset C_{\dot{T}_{Y}^{*} X}^{1}(\mathcal{M}) .
\end{aligned}
$$

Therefore b") holds provided that we prove (8).

Let $\theta \in T_{V}\left(T^{*} X\right)$ such that $\theta \notin C_{V}^{1}(\mathcal{M})$. Considering the local coordinates in $T_{V}\left(T^{*} X\right),\left((x, t ; \eta) ; \tilde{\xi}_{1}, \cdots, \tilde{\xi}_{n-1}\right)$ and using the technique of [MF2] or [S], we may assume $\theta=((0,0 ; 1) ; 1, \cdots, 0)$. We shall identify $T_{Y}^{*} X \cap\{\zeta=1\}$ to $Y$. Furthermore, we may assume by classical arguments that $\mathcal{M}$ is of the form $\mathcal{E}_{X} / \mathcal{E}_{X} P$ with

$$
P\left(x, t, D_{x}, D_{t}\right)=D_{x_{1}}^{m}+\sum_{0 \leq j \leq m-1} A_{j}\left(x, t, D_{x^{\prime}}, D_{t}\right) D_{x_{1}}^{j}
$$

where $D_{x^{\prime}}=\left(D_{x_{2}}, \cdots, D_{x_{n-1}}\right)$ and $A_{j} \in \mathcal{E}_{V}(m-j)$. We shall prove that $\left(0, d x_{1}\right) \notin S S\left(\left.R \mathcal{H} \operatorname{om}_{\mathcal{E}_{X}}\left(\mathcal{M}, C_{Y \mid X}^{\mathbb{R}, f}\right)\right|_{\{\zeta=1\}}\right)$. For that purpose we need Lemmas 2.5, 2.6 and 2.7 below.

Lemma 2.5. The sheaf $C_{Y \mid X}^{\mathbb{R}, f}$ satisfies:

1) The analytic continuation principle: Let $\omega \subset \Omega$ be two open subsets of $Y, \Omega$ connected and $\omega \neq \phi$, and assume that $u \in \Gamma\left(\Omega, C_{Y \mid X}^{\mathbb{R}, f}\right)_{\{\zeta=1\}}$ vanishes in $\omega$. Then $u \equiv 0$.

2) If $V$ is a conic subset of $\dot{T}_{Y}^{*} X$ of the form $W \times \Gamma$, where $W$ is compact Stein in $Y$ and $\Gamma$ a convex cone in $\mathbb{C}^{*}$ containing 1 , such that $\Gamma \cap S^{1}$ is closed, then,

$$
\forall j \geq 1, \quad H^{j}\left(V, C_{Y \mid X}^{\mathbb{R}, f}\right)=0 .
$$

In particular, if $W$ is compact Stein in $Y, H^{j}\left(W,\left.C_{Y \mid X}^{\mathbb{R}, f}\right|_{\{\zeta=1\}}\right)=0, \forall j \geq 1$.

Proof. 1) Since $C_{Y \mid X}^{\mathbb{R}, f}$ is a subsheaf of $C_{Y \mid X}^{\mathbb{R}}$, it is enough to prove that $u$ vanishes as a section of $\left.C_{Y \mid X}^{\mathbb{R}}\right|_{\{\zeta=1\}}$ but this is a consequence of the analytic 
continuation principle for $C_{Y \mid X}^{\mathbb{R}}$ which is well known (cf. [K-S1]).

2) We have

$$
\forall j, \quad H^{j}\left(W \times \Gamma, C_{Y \mid X}^{\mathbb{R}, f}\right)=\lim _{\overrightarrow{W^{\prime}, \Gamma^{\prime}}} H^{j}\left(W^{\prime} \times \Gamma^{\prime}, C_{Y \mid X}^{\mathbb{R}, f}\right),
$$

where $W^{\prime}$ runs through a neighborhood system of $W$ formed by open Stein relatively compact subsets of $Y$ and $\Gamma^{\prime}$ through a neighborhood system of $\Gamma$ formed by convex open cones.

Let $V^{\prime}=W^{\prime} \times \Gamma^{\prime}$. Then

$$
H^{j}\left(V^{\prime}, C_{Y \mid X}^{\mathbb{R}, f}\right) \simeq H_{V^{\prime 0}}^{j+1}\left(\tau^{-1} \pi\left(V^{\prime}\right),{ }^{t} \nu_{Y}\left(\mathcal{O}_{X}\right)\right)
$$

where $\tau: T_{Y} X \rightarrow Y$ is the projection and ${ }^{t} \nu_{Y}\left(\mathcal{O}_{X}\right)$ denotes the tempered specialisation of the sheaf $\mathcal{O}_{X}$ along $Y$. We denote by $\nu_{Y}\left(\mathcal{O}_{X}\right)$ the usual specialisation of $\left(\mathcal{O}_{X}\right)$ along $Y$. Let us study the long exact sequence

$$
\begin{aligned}
(10) \cdots & \rightarrow H_{V^{\prime} 0}^{i}\left(\tau^{-1} \pi\left(V^{\prime}\right),{ }^{t} \nu_{Y}\left(\mathcal{O}_{X}\right)\right) \rightarrow H^{i}\left(\tau^{-1} \pi\left(V^{\prime}\right),{ }^{t} \nu_{Y}\left(\mathcal{O}_{X}\right)\right) \rightarrow \\
& \rightarrow H^{i}\left(\tau^{-1} \pi\left(V^{\prime}\right) \backslash V^{\prime o},{ }^{t} \nu_{Y}\left(\mathcal{O}_{X}\right)\right) \rightarrow H_{V^{\prime}}^{i+1}\left(\tau^{-1} \pi\left(V^{\prime}\right),{ }^{t} \nu_{Y}\left(\mathcal{O}_{X}\right)\right) \rightarrow \cdots
\end{aligned}
$$

On one hand, we have, $\forall i \geq 1$ :

$$
\begin{aligned}
H^{i}\left(\tau^{-1} \pi\left(V^{\prime}\right),{ }^{t} \nu_{Y}\left(\mathcal{O}_{X}\right)\right) & =H^{i}\left(\tau^{-1} \pi\left(V^{\prime}\right), \nu_{Y}\left(\mathcal{O}_{X}\right)\right) \\
& =H^{i}\left(W^{\prime}, \mathcal{O}_{X}\right) \\
& =0
\end{aligned}
$$

On the other hand, we have $V^{\prime \circ}=W^{\prime} \times \Gamma^{\prime \circ}$, therefore $\tau^{-1} \pi\left(V^{\prime}\right) \backslash V^{\prime \circ}=$ $W^{\prime} \times\left(\mathbb{C} \backslash \Gamma^{\prime \circ}\right)$.

Hence it remains to prove that, $\forall i \geq 1$,

$$
\lim _{\substack{W^{\prime}, \Gamma^{\prime}}} H^{i}\left(W^{\prime} \times\left(\mathbb{C} \backslash \Gamma^{\prime \circ}\right),{ }^{t} \nu_{Y}\left(\mathcal{O}_{X}\right)\right)=0
$$

This direct limit equals

$$
H^{i}\left(W \times H,{ }^{t} \nu_{Y}\left(\mathcal{O}_{X}\right)\right)=0
$$

where $H$ is the cone generated by $\overline{\mathbb{C} \backslash \Gamma^{\circ}} \cap S^{1}$. Let now $V$ denote $W \times H$.

We have

$$
H^{i}\left(V,{ }^{t} \nu_{Y}\left(\mathcal{O}_{X}\right)\right)=\lim _{\vec{U}} H^{i}\left(\mathbb{R} \Gamma\left(X, t \mathcal{H o m}\left(\mathbb{C}_{U}, \mathcal{O}_{X}\right)\right),\right.
$$


with $U$ running through the open subanalytic sets in $X$ such that $V \cap C_{Y}(X \backslash$ $U)=\phi$, by $(3.1 .2)$ of $[\mathrm{A}]$.

By Proposition 4.1.3 of [K-S3], $U$ may be taken to range through the family $p\left(U^{\prime} \cap t^{-1}\left(\mathbb{R}^{+}\right)\right)$where $U^{\prime}$ ranges through a neighborhood system of $V$ in the real normal deformation of $X$ along $Y, \tilde{X}_{Y}, t: \tilde{X}_{Y} \rightarrow \mathbb{R}$ being the canonical projection and $p: \tilde{X}_{Y} \rightarrow X$ the deformation morphism. Since $V=W \times H$ we may assume that $U$ is of the form $\left(W^{\prime} \times \Gamma^{\prime \prime}\right) \cap B$ where $W^{\prime}$ is Stein subanalytic, $\Gamma^{\prime \prime}$ is an open cone in $\mathbb{C}$ and $B$ is an open polydisc in $\mathbb{C}^{n}$. Hence $U$ is Stein subanalytic relatively compact and we may apply Hörmander's results in $[\mathrm{H}]$. More precisely, $t \mathcal{H} \mathrm{om}\left(\mathbb{C}_{U}, \mathcal{O}_{X}\right)$ is concentrated in degree zero and $H^{i}\left(X, t \mathcal{H o m}\left(\mathbb{\mathbb { C }}_{U}, \mathcal{O}_{X}\right)\right)=0, \forall i>0$. (Cf. also Lemma 2.6 and Lemma 2.16 of $[\mathrm{Be}]$.

Proof of Lemma 2.4 (continued). We shall now use some concepts introduced by J.M. Bony and P. Schapira (cf. [B-S]).

Let $\Omega$ be an open convex subset of $Y$. Let us note $H_{h}$ and $Z$, respectively, the hyperplane of $\mathbb{C}^{n}$ of equation $x_{1}=h$ and $x_{1}=0$, hence $Z=H_{0}$. Let $\delta$ be a real positif number. We say that $\Omega$ is $\delta$-Y $\cap H_{h}$-flat if, whenever $x \in \Omega$ and $\tilde{x} \in H_{h}$, satisfy

$$
\left|x_{1}-h\right| \geq \delta\left|x_{j}-\tilde{x}_{j}\right|_{j=2, \cdots, n-1}
$$

entails $\tilde{x} \in \Omega$.

If $\Omega$ is $\delta-Y \cap Z$-flat, then for any $\rho>0, \rho \Omega$ is still $\delta-Z \cap Y$-flat, and for any $\omega=(\varepsilon, 0, \cdots, 0), \Omega+\omega$ is $\delta$ - $(Z+\omega) \cap Y$-flat.

Let $P\left(x, t, D_{x}, D_{t}\right)$ be of the form (9), that is, $P$ is Weierstrass with respect to $D_{x_{1}}$, and belongs to $\mathcal{E}_{V}$ in a suitable neighborhood of $(0 ; 1, \cdots, 0)$.

Lemma 2.6 (Precised Cauchy Problem). There exists an open neighborhood $\Omega_{1}$ of $0 \in Y$ and $\delta>0$ such that, for any convex open subset $\Omega \subset \Omega_{1}$

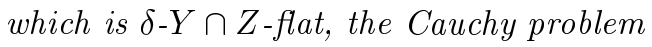

$$
P_{f}=g, \quad \gamma(f)=(h)
$$

where $\gamma(f)=\left(\left.f\right|_{Z}, \cdots,\left.D_{x_{1}}^{m-1} f\right|_{Z}\right),\left.g \in C_{Y \mid X}^{\mathbb{R}, f}\right|_{\{\zeta=1\}}(\Omega)$ and

$$
\left.(h) \in C_{Y \cap Z \mid Z}^{\mathbb{R}, f}\right|_{\{\zeta=1\}}(\Omega \cap Z)^{m}
$$

admits a unique solution

$$
\left.f \in C_{Y \mid X}^{\mathbb{R}, f}\right|_{\{\zeta=1\}}(\Omega) .
$$


Moreover, there exists $h_{0}>0$ depending only on $P, \Omega_{1}$ and $\delta$, such that, if $\Omega$ is $\delta$ - $H_{h} \cap Y$-flat, and the Cauchy data is given on $H_{h}$ for $|h|<h_{0}$, the same result holds in $\Omega$.

We shall not give here the detailed proof of this lemma since the unicity is an immediate consequence of Lemma 5.2 of [K-S2] and, as for the existence, one follows step by step the proof of this same lemma, using Theorem 2.4.3 of [B] to be sure that the unique solution is in fact in $C_{Y \mid X}^{\mathbb{R}, f}$.

We give here a version, in our framework, of Zerner's classical result on the propagation at the boundary for holomorphic solutions of partial differential equations $([\mathrm{Z}])$.

Lemma 2.7. Let $\varphi$ be a $C^{\infty}$ function in a neighborhood of $0 \in Y$ such that $\varphi(0)=0$ and $d \varphi(0)=d x_{1}$. Let $\Omega=\{x, \varphi(x)<0\}$. Let $u \in$ $\Gamma\left(\Omega,\left.C_{Y \mid X}^{\mathbb{R}, f}\right|_{\{\zeta=1\}}\right)$ and assume that $P u$ extends as a section of $\left.C_{Y \mid X}^{\mathbb{R}, f}\right|_{\{\zeta=1\}}$ to a neighborhood of 0 . Then $u$ extends to a neighborhood of 0 .

Proof. Let $\Omega_{1}, h_{0}$ and $\delta$ be as in Lemma 2.6. We may assume $\varphi$ is defined in $\Omega_{1}$, and that $P u$ extends to $\Omega_{1}$. We have $\varphi(x)=\operatorname{Re} x_{1}-\psi\left(\operatorname{Im} x_{1}, x_{2}, \cdots\right.$, $\left.x_{n-1}\right)$ with $d \psi(0)=0$. Let $0<\varepsilon \ll 1$ : there exists $R>0$ such that $\left\|x^{\prime}\right\|<R$ entails $-\varepsilon<\psi\left(0, x^{\prime}\right)$, that is, denoting as before $H_{-\varepsilon}=\left\{x_{1}=-\varepsilon\right\}$,

$$
H_{-\varepsilon} \cap\left\{\left(x, x^{\prime}\right) \in \Omega_{1},\left|x^{\prime}\right|<R\right\} \subset \Omega .
$$

Since $\psi\left(0, x^{\prime}\right)=0\left(\left|x^{\prime}\right|\right)$, we may assume that $\varepsilon<\delta R$ and that the open polydisc centered in $(-\varepsilon, 0,0)$ with radius $\max (R, \delta R)$ is contained in $\Omega_{1}$.

Then $W_{\varepsilon}=\left\{\left(x_{1}, x^{\prime}\right) ;\left|x_{1}+\varepsilon\right|<\delta\left(R-\left\|x^{\prime}\right\|\right),\left\|x^{\prime}\right\|<R\right\} \subset \Omega_{1}$ will be $\delta$ - $H_{-\varepsilon} \cap Y$-flat and is a neighborhood of zero.

Let us now consider the Cauchy problem :

$$
P u_{\varepsilon}=P u, \quad \gamma_{\varepsilon}\left(u_{\varepsilon}\right)=\gamma_{\varepsilon}(u)
$$

where $\gamma_{\varepsilon}$ denotes the traces along $H_{-\varepsilon}$. By Lemma 2.6, the solution $u_{\varepsilon}=u$ is defined in $W_{\varepsilon}$, which achieves the proof.

Conclusion of the proof of Lemma 2.4 and of Theorem 2.1. We shall use Proposition 5.1.1. 3) of [K-S3] which gives a characterization of the microsupport of a complex of sheaves better adapted to our situation.

Let $p_{0}=\left(0 ; d x_{1}\right) \in T^{*} Y$. Let $\Omega_{1}, \delta$ and $h_{0}$ be given by Lemma 2.6. Let $0<\varepsilon \ll h_{0}$ and $0<R \ll 1$ be small enough and satisfying : 
i) $\varepsilon<R$,

ii) the open polydisc $B_{R}(-\varepsilon)$ centered in $(-\varepsilon, 0, \cdots 0)$ and radius $R$ is contained in $\Omega_{1}$.

Let $U=B_{R}(-\varepsilon)$. Let $\gamma$ be the proper closed convex cone of $\mathbb{C}^{n-1}$ defined by

$$
\gamma=\left\{\left(x_{1}, \cdots, x_{n-1}\right), \operatorname{Re} x_{1} \leq-\delta\left(\| x^{\prime}||+\left|\operatorname{Im} x_{1}\right|\right)\right\} .
$$

In particular, $(1,0 \cdots 0) \in \operatorname{int} \gamma^{\circ a}, \underline{a}$ denoting the antipodal map.

It is easy to check that $(U+\gamma) \cap H$, where $H$ denotes the real half space $\left\{\left(x_{1}, x^{\prime}\right)\right.$, Re $\left.x_{1} \geq-\varepsilon\right\}$, is bounded. Hence, for any $x \in U, H \cap(x+\gamma)$ is a compact.

Up to a suitable choice of $\varepsilon, R$ and $\delta$, we may assume that $(U+\gamma) \cap H \subset \Omega_{1}$ and

$$
\{(U+\gamma) \cap H\} \times \operatorname{Int} \gamma^{\circ a} \cap C_{V}^{1}\left(\frac{\mathcal{E}_{X}}{\mathcal{E}_{X} P}\right)=\phi .
$$

Let $L=\left\{x, \operatorname{Re} x_{1}=-\varepsilon\right\}$. We shall prove that the natural morphism of complexes :

$$
\begin{gathered}
R \Gamma\left(H \cap(x+\gamma),\left.C_{Y \mid X}^{\mathbb{R}, f}\right|_{\{\zeta=1\}}\right) \stackrel{P}{\rightarrow} R \Gamma\left(H \cap(x+\gamma),\left.C_{Y \mid X}^{\mathbb{R}, f}\right|_{\{\zeta=1\}}\right) \\
\downarrow \\
\downarrow \\
R \Gamma\left(L \cap(x+\gamma),\left.C_{Y \mid X}^{\mathbb{R}, f}\right|_{\{\zeta=1\}}\right) \stackrel{P}{\rightarrow} R \Gamma\left(L \cap(x+\gamma),\left.C_{Y \mid X}^{\mathbb{R}, f}\right|_{\{\zeta=1\}}\right)
\end{gathered}
$$

is a quasi isomorphism.

Since $H \cap(x+\gamma)$ and $L \cap(x+\gamma)$ are compact convex subsets, hence Stein, by Lemma 2.5 it remains to prove that $P$ induces an isomorphism in the quotient

$$
\frac{\Gamma\left(L \cap(x+\gamma),\left.C_{Y \mid X}^{\mathbb{R}, f}\right|_{\{\zeta=1\}}\right)}{\Gamma\left(H \cap(x+\gamma),\left.C_{Y \mid X}^{\mathbb{R}, f}\right|_{\{\zeta=1\}}\right)} .
$$

Here, we used the analytic continuation principle to identify $\Gamma\left(H \cap(x+\gamma),\left.C_{Y \mid X}^{\mathbb{R}, f}\right|_{\{\zeta=1\}}\right)$ with a submodule of $\Gamma\left(L \cap(x+\gamma),\left.C_{Y \mid X}^{\mathbb{R}, f}\right|_{\{\zeta=1\}}\right)$.

To prove the surjectivity of $P$, we apply the analogous of Lemma 2.4.7 of [MF1] or Lemma 3.1.5 of [S] which is proved by the same method thanks to Lemmas 2.6 and 2.7. Let $v \in \Gamma\left(L \cap(x+\gamma),\left.C_{Y \mid X}^{\mathbb{R}, f}\right|_{\{\zeta=1\}}\right)$. We solve the equation $P u=v$ in a neighborhood of $H_{-\varepsilon} \cap(x+\gamma)$ using Lemma 2.6 and then extend $u$ to a neighborhood of $L \cap(x+\gamma)$ since any real hyperplane through $x$, with a 1-microcharacteristic normal, which intersects $L \cap(x+\gamma)$ intersects $H_{-\varepsilon} \cap(x+\gamma)$ (see for example page 152 of $[\mathrm{S}]$ ).

As for the injectivity, we shall use the construction of [K-S3, Proposition 5.1.5]. 
For each $a \in U \cap(H \backslash L)$, we construct a family of open subsets $\left\{\Omega_{t}(a)\right\}_{t \in \mathbb{R}^{+}}$, such that :

i) $\Omega_{t}(a) \subset a+$ int $\gamma$,

ii) $\Omega_{t}(a) \cap L=(a+$ int $\gamma) \cap L$,

iii) $\Omega_{t}(a)=\underset{r<t}{\cup} \Omega_{r}(a)$,

iv) $\delta \Omega_{t}(a)$ is smooth real analytic,

v) $Z_{t}(a)=\left(\underset{s>t}{\cap} \overline{\Omega_{s}(a) \backslash \Omega_{t}(a)}\right) \cap H \subset \delta \Omega_{t}(a)$, and the conormal of $\Omega_{t}(a)$ at $Z_{t}(a)$ is non 1-microcharacteristic for the operator $P$ everywhere in $Z_{t}(a)$,

vi) $\left(\cup_{t>0} \Omega_{t}(a)\right) \cap H=(a+\operatorname{int} \gamma) \cap H$,

vii) $\left(\underset{t>0}{\cap} \Omega_{t}(a)\right) \cap H=(a+\operatorname{int} \gamma) \cap L$.

We recall that if $v=(1,0, \cdots 0)$, then the family

$$
\{(x+\rho v+\operatorname{int} \gamma) \cap H\}_{\rho>0}
$$

forms a neighborhood system of $(x+\gamma) \cap H$, and the family $\left\{\Omega_{t}(x+\rho v) \cap\right.$ $H\}_{\rho>0, t>0}$ forms a neighborhood system of $(x+\gamma) \cap L$.

Let $u \in \Gamma\left(L \cap(x+\gamma),\left.C_{Y \mid X}^{\mathbb{R}, f}\right|_{\{\zeta=1\}}\right)$ such that $P u=w$ extends to a neighborhood of $H \cap(x+\gamma)$.

Let $\rho>0$ and $t_{0}>0$ such that $u$ is defined in $\Omega_{t_{0}}(x+\rho v) \cap H$ and $w$ in $(x+\rho v+\operatorname{int} \gamma) \cap H$. By v) and Lemma 2.7, $u$ extends to a neighborhood of $\delta \Omega_{t_{0}}(x+\rho v) \cap H$ hence to $\Omega_{t^{\prime}}(x+\rho v) \cap H$ for some $t^{\prime}>t_{0}$, and the definition of the family $\Omega_{t}$ entails that this procedure leads to an extension of $u$ in a neighborhood of $(x+\gamma) \cap H$.

Remark. By the functorial properties of $\mu \mathrm{hom}\left(\cdot, \mathcal{O}_{X}\right)$, assuming that $\mathcal{M}$ is generated by a coherent $\mathcal{D}_{X}$-module, one easily deduces that if $F$ is an object of $D_{\mathbb{R}-c}^{b}(X)$ such that $S S(F) \subset V$ as in Theorem 2.1,

$$
S S\left(\mathbb{R} \mathcal{H} \operatorname{om}_{\mathcal{E}_{X}}\left(\mathcal{M}, \mu \operatorname{hom}\left(F, \mathcal{O}_{X}\right)\right)\right) \subset C_{V}(\mathcal{M}),
$$

and this inclusion may still be improved to the microdifferential framework using other tools.

Our conjecture is that Theorem 2.1 may be generalized to the case where $F \in D_{\mathbb{R}-c}^{b}(X)$. 


\section{References}

[A] Andronikof, E., Microlocalisation tempérée, supplément au Bull. Soc. Math. France, mémoire $\mathrm{n}^{\circ}$ 57, 122, No.2 (1994).

[B] Bony, J-M., Propagation des singularités différentiables pour une classe d'opérateurs différentiels à coefficients analytiques, Astérisque, 34-35 (1976), 43-92.

[B-S] Bony, J-M. and Schapira, P., Propagation des singularités analytiques pour les solutions des équations aux dérivées partielles, Ann. Inst. Fourier, 26 (1976), 81-140.

[Be] Berni, O., A vanishing theorem for formal cohomology of perverse sheaves, J. Funct. Anal., 158 (1998), 2, 267-288

[H] Hörmander, L., An introduction to Complex Analysis in several variables, Van Nostrand, Princeton, NJ, 1966.

[K] Kashiwara, M., The Riemann-Hilbert problem for holonomic systems, Publ. RIMS, Kyoto Univ., 20 (1984), 319-365.

[K-O] Kashiwara, M. and Oshima, T., Systems of differential equations with regular singularities and their boundary value problems, Ann. of Math., 106 (1977), 145-200.

[K-S1] Kashiwara, M. and Schapira, P., Microhyperbolic systems, Acta Math., 142 (1979), $1-55$.

[K-S2] — - Problème de Cauchy pour les systèmes microdifférentiels dans le domaine complexe, Invent. Math., 46 (1978), 17-38.

[K-S3] — Sheaves on manifolds, Grundlehren Math. Wiss, 292, Springer Verlag, 1990.

[K-S4] - Microlocal study of sheaves, Astérisque, 128 (1985).

[K-S5] - Moderate and formal cohomology associated with constructible sheaves, Mém. Soc. Math. Fr., 94 (1996).

[K-S6] - Ind-sheaves, The RIMS prepublications, (1999).

[L] Laurent, Y., Théorie de la deuxième microlocalisation, Progr. Math., Birkhäuser, 53 (1985).

[MF1] Monteiro Fernandes, T., Problème de Cauchy pour les systèmes microdifférentiels, Astérisque, 140-141 (1986), 135-220.

[MF2] — - Propagation et constructibilité pour les systèmes microdifférentiels formels, Astérisque, 140-141 (1986), 221-250.

[MF3] — Formal and tempered solutions of regular $\mathcal{D}$-modules, Compositio Math. to appear.

[S] Schapira, P., Microdifferential systems in the complex domain, Grundlehren Math. Wiss., 269, Springer Verlag, 1985.

[S-K-K] Sato, M., Kawai, T. and Kashiwara, M., Hyperfunctions and pseudodifferential equations, Lecture Notes in Math., Springer, 287 (1973), 265-529.

[Z] Zerner, M., Domaine d'holomorphie des fonctions vérifiant une équation aux dérivées partielles, C.R.Acad. Sci. Paris Sér. I Math., 272 (1979), 1646-1648. 